\title{
Inhibited Metastasis and Amplified Chemotherapeutic Effects by Epigene-Transfection Based on a Tumor-Targeting Nanoparticle
}

This article was published in the following Dove Press journal: International Journal of Nanomedicine

\author{
Mengzhu Wang' \\ Zhigang Wang (D) \\ Bin Qiao (ID) \\ Jin $\mathrm{Cao}$ \\ Luya Quan (D) \\ Yuanli Luo' \\ Hanwen $\mathrm{Qi}^{2}$ \\ Xiaowen Zhong $\mathbb{D}^{\prime}$ \\ Yubei He $\mathbb{D}^{\prime}$ \\ Xianquan Zhang ${ }^{1,3}$ \\ Lan $\mathrm{Hao}^{\prime}$
}

'Institute of Ultrasound Imaging, The Second Affiliated Hospital of Chongqing Medical University, Chongqing 400010 , People's Republic of China; ${ }^{2}$ The A. Gary Anderson Graduate School of Management, University of California, Riverside, Riverside, CA, 9252I, USA; ${ }^{3}$ Cardiothoracic Surgery Department, Chongqing Hygeia Cancer Hospital, Chongqing, 40I33I, People's Republic of China

Correspondence: Lan Hao Institute of Ultrasound Imaging, The Second Affiliated Hospital of Chongqing Medical University, I\# Yixueyuan Road, Yuzhong District, Chongqing, People's Republic of China

Tel +86 I582329l038

Email lanhao5@cqmu.edu.cn

Xianquan Zhang

Chongqing Hygeia Cancer Hospital,No.

222, Sixian Road, University Town,

Shapingba District, Chongqing 40I33I,

People's Republic of China

Tel +86 I3996286/25

Email xqzhng@।63.com
Purpose: Tumor metastasis and drug resistance have always been vital aspects to cancer mortality and prognosis. To compromise metastasis and drug resistance, a nanoparticle IPPDPHF2 (IR780/PLGA-PEI(Dox)-PHF2) has been engineered to accomplish efficient targeted epigenotherapy forced by PHF2-induced MET (mesenchymal to epithelial transition).

Materials and Methods: IPPD-PHF2 nanoparticle was synthesized and characterized by several analytical techniques. The transfection efficiency of IPP-PHF2 (IR780/PLGA-PEIPHF2) was compared with PP-PHF2 (PLGA-PEI-PHF2) in vitro by WB and in vivo by IHC, and the cytotoxicity of IPP was compared with Lipo2000 in vitro by CCK 8 assay. The inhibition of cancer cell migration caused by PHF2-upregulation was tested by wound healing assay, and the enhanced chemotherapeutic sensitivity was detected by flow cytometry. Tumor-targeting property of IPPD-PHF2 was proved by fluorescent imaging in vivo with MDA-MB-231 tumor-bearing nude mice. Except for fluorescent imaging ability, considerable photoacoustic signals of IPPD-PHF2 at tumor sites were verified. The anti-tumor activity of IPPD-PHF2 was investigated using in vivo human breast cancer MDA-MB-231 cell models.

Results: Tumor-targeting nanoparticle IPPD-PHF2 had an average size of about $319.2 \mathrm{~nm}$, a stable zeta potential at about $38 \mathrm{mV}$. The encapsulation efficiency of doxorubicin was around $39.28 \%$, and the adsorption capacity of plasmids was about $64.804 \mu \mathrm{g} / \mathrm{mg}$. Significant up-regulation of PHF2 induced MET and caused reduced migration as well as enhanced chemotherapeutic sensitivity. Either IPPD (IR780/PLGA-PEI(Dox)) or IPP-PHF2 (IR780/ PLGA-PEI-PHF2) presented minor therapeutic effects, whereas IPPD-PHF2 specifically accumulated within tumors, showed extraordinary transfection efficiency specifically in tumor sites, acted as inhibitors of metastasis and proliferation, and presented good multimodality imaging potentials in vivo.

Conclusion: IPPD-PHF2 NPs is a promising tool to bring epigenotherapy into a more practical era, and the potential application of harm-free multimodality imaging guidance is of great value.

Keywords: targeted epigenotherapy, metastasis, drug resistance, multimodality imaging, breast cancer

\section{Introduction}

Epigenetic regulations such as DNA ethylating, chromatin accessibility, histone tail modification are strongly interlinked to crucial cellular processes, whereas their dysregulation may cause aberrant gene expression, by which tumors slide in. ${ }^{1}$ Plant homeodomain finger 2 (PHF2) belongs to the JumonjiC family, which contains 
a JumonjiC domain and a plant homeodomain finger, and has an important role in epigenetic regulation of gene expression. PHF2 gene is frequently deleted in several cancers. It has been identified to convert the transcription activity via demethylating the repressive $\mathrm{H} 3 \mathrm{~K} 9 \mathrm{me} 2 / 3$ mark, which may contribute to the reduced drug resistance and tumor metastasis directly or indirectly. ${ }^{2-5}$ Several studies have explored wide-ranged signal pathways related to PHF2, including PHF2-p53, PHF2-PHF8, PKA-cAMPPHF2 and others which are yet to be uncovered. ${ }^{6-9}$ EMT (epithelial-to-mesenchymal-transition) has been clarified as a set of multiple and dynamic transitional states between epithelial and mesenchymal phenotypes. EMT and its intermediate states have been proved to be tightly linked to tumor progression. ${ }^{10,11}$ Activation of PKA can induce MET (mesenchymal-to-epithelial-transition) in mesenchymal human mammary epithelial cells. PHF2, as a PKA substrate, facilitates TICs (tumor initial cells) to differentiate into a more epithelial state, lose tumorinitiating ability and become more vulnerable to traditional chemotherapy. $^{7}$

The anti-tumor function of p53 is strictly regulated by upstream and downstream influence factors. ERK2 is an important upstream kinas of p53 activation; it can phosphorylate $\mathrm{p} 53$ on Thr55. Meanwhile, it can be activated by Dox (doxorubicin). In addition, $\mathrm{p} 21$, as a downstream product of $\mathrm{p} 53$, undertakes subsequent $\mathrm{p} 53$ anticancer functions. PHF2 is necessary for inducing p21, and it guarantees $\mathrm{p} 53$-associated cell apoptosis in response to chemotherapy. 6,12

The intimate crosstalk between PHF2 and Doxorubicin inspired us to propose this new combined treatment on breast cancer: epigenotherapy by PHF2-overexpression and amplified chemotherapy by Doxorubicin (Figure 1B).

To achieve the precise and efficient gene transfection in vitro and in vivo, nanoparticle has emerged as a sound vector for drug delivery and gene transfection. ${ }^{13}$ Unlike the transfection based on viral system which may cause safety issues due to the immunogenicity, PLGA is one of the FDAapproved materials. PLGA nanoparticles can avoid attack from endo-lysosome and thus protect the cargo from degradation or early-release. ${ }^{14-17}$ Basically, two kinds of methods have been reported to load plasmids to nanoparticles-Physical entrapment and electrostatic adsorption. For physical entrapment, limited entrapment efficiency, low release efficiency, and DNA-ring-opening/DNA break damage caused by physical/chemical actions during the synthetic process are main limitations for effective transfection.
PLGA protected plasmids from degradation and structure/ function damages caused by bioactive enzymes, however, given the limited encapsulation capacity and structure damage, the transfection efficiency is unsatisfying. ${ }^{18}$ Electrostatic adsorption comes up as a better choice for transfection. PEI, given its adhesive properties, chemical stability and stable electrical property, has become a considerable material for cationic nanoparticle modification. ${ }^{19}$ Simply blending branched PEI with PLGA or PLGA nanoparticle have shortcomings, such as unstable adsorption and early release, which may cause low transfection efficiency or curtate gene expression period. ${ }^{20}$ Previous study has reported a successful implementation of covalent conjugation between branched PEI and PLGA, which is theoretically based on the formation of peptide bond via carbodiimide reaction. ${ }^{19}$ However, not only should the transfection efficiency be focused, but also the transfection accuracy needs to be taken into account. Gene transfection efficiency can be significantly improved by feat of the nanoparticle possessing active-targeting ability rather than the one owning only the passive-targeting ability based on tumor-enhanced permeability and retention effect (EPR effect) which also in a way helps with nanoparticles accumulation.

IR780 iodide belongs to heptamethine dyes which exhibits a peak spectral absorption at $780 \mathrm{~nm}$, and it has been rising up as a practical material in theranostics due to the tumormitochondria-targeting ability, fluorescence and deep tissue penetration. $^{21-24}$ Given that, IR780 iodide was embedded into PLGA nanoparticles. Furthermore, Doxorubicin $\cdot \mathrm{HCl}$ was added inside regarding the crosstalk effects in intracellular signaling pathways.

Comparing to PP (PLGA-PEI nanoparticles) and transfection kit on the market, IPP (IR780/PLGA-PEI) possesses following key advantages: 1. Precise localization of tumor sites. 2. Harm-free multimodality imaging. 3. Enhanced intracellular uptake. 4. Less cytotoxicity. 5. Common application in vitro and in vivo, which means it may become a potential tool for future application in clinic. With all these superiorities, IPPD-PHF2 (IR780/ PLGA-PEI (Dox)-PHF2) nanoparticles displayed noticeable suppression effect in breast tumor growth and metastasis both in vitro and in vivo, and the whole treating process is displayed in Figure 1.

\section{Materials and Methods}

\section{Reagents and Materials}

Resomer RG502H Poly (D,L-lactide-co-glycolide, acid terminated), Polyethylenimine (branched, Mw 25000), Poly (vinyl 


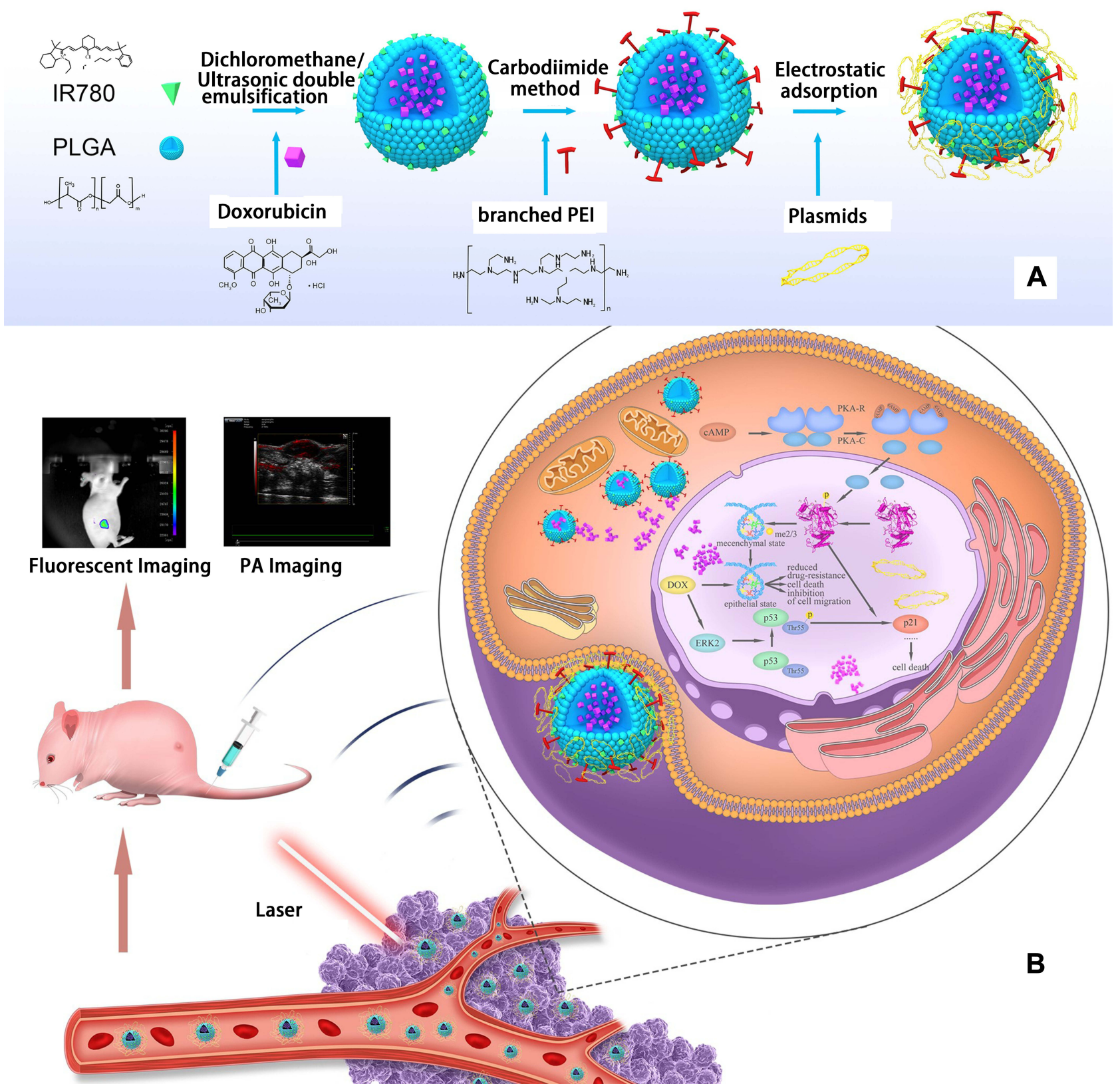

Figure I The synthesis and therapeutic process.

Notes: (A) The synthesis procedure of IPPD-PHF2. (B) Tumor-targeting gene-drug-combined PLGA nanoparticle, termed as IPPD-PHF2, was engineered to integrate epigenetic regulation and chemotherapy to induce MET, amplify the inhibition of tumor metastasis and proliferation, and work as a potential PA/FL imaging probe.

alcohol), Ethylcarbodiimide hydrochloride were purchased from Sigma Aldrich (product of Germany). Sulfo-NHS was purchased from Thermo Fisher Scientific (USA). Lipofectamine 2000 Reagent was purchased from Invitrogen (Thermo Fisher Scientific, USA). IR780 iodide was purchased from Alfa Aesar (product of Ukraine). EndoFree Maxi Plasmid Kit was purchased from TIANGEN BIOTECH Co. Ltd (Beijing, China). PHF2-pCDNA3.1-EGFP-Amp plasmid was constructed in TINGJUNTECH (Thermo Fisher
Scientific, Shanghai, China). Isopropyl alcohol was purchased from MACKLIN Biochemical Co. Ltd (Shanghai, China). Cell Counting Kit 8 (CCK8), Gel-Red, DAPI, DiI were purchased from Beyotime Technology. Doxorubicin hydrochloride was purchased from Adamas Reagent Co, Ltd. (Shanghai, China). PHF2 primary antibody was purchased from Cell Signaling Technology (USA). E-cadherin/Vimentin/ER $\alpha /$ CX43 primary antibody were purchased from ServiceBio (Wuhan, China). 


\section{Synthesis}

IPPD (IR780/PLGA-PEI (Dox)) NP was synthesized through ultrasonic double emulsification method followed with carbodiimide method. The whole process is illustrated in Figure 1A. Firstly, IR780 (2mg) and PLGA-COOH $(50 \mathrm{mg})$ were dissolved in dichloromethane $(1 \mathrm{~mL})$, Doxorubicin Hydrochloride (3mg) was diluted in doublestilled water (200ul), then Dox solution (200ul) was added into the former solution as the inner aqueous phase for the first ultrasonic emulsification with an ultrasonic probe (Vibra cell 54175T, Sonics \& Materials Inc., USA) (120kW, an interval of 5 seconds, 2 min $40 \mathrm{~s})$. Secondly, PVA $(4 \mathrm{~mL}, 40 \mathrm{~g} / \mathrm{L})$ was added as the outer aqueous phase for the second ultrasonic emulsification (same arguments as before except for $2 \min 20$ s of emulsification time). After that isopropanol solution $(6 \mathrm{~mL}, 20 \mathrm{~mL} / \mathrm{L})$ was blended in, and the mixture was magnetic stirred for 3 hours to evaporate dichloromethane. The mixture was centrifugated at $10500 \mathrm{rpm}$ for $8 \mathrm{~min}$ (TG18, Ping fan Centrifuge Company, Changsha, China) and washed by MES solution $(0.1 \mathrm{M}, \mathrm{pH}=5.0)$ for twice. The final sediment was resuspended in MES $(1 \mathrm{~mL})$ solution and prepared for the covalent conjugation between PLGA and branched PEI by carbodiimide reaction. EDC (100mg) and sulfo-NHS (30mg) were dissolved in MES solution ( $1 \mathrm{~mL}$ ) in ice bath, and nanoparticle suspension was dropwise added in, and this mixture was gently magnetically stirred for $2 \mathrm{~h}$ at room temperature. Branched PEI (3g) was dissolved in MES solution $(10 \mathrm{~mL})$ under the help of ultrasonic cleaner. Branched PEI solution was blended with the former mixture and gently magnetically stirred for another $2 \mathrm{~h}$. After the reaction, the ultimate suspension was diluted with equal amount of double-stilled water. Then it was transferred to $2 \mathrm{~mL}$ EP tubes and centrifuged at the speed of 12000rpm for 8 min (HERAEUS PICO17 Centrifuge, Thermo Fisher). The sediments were collected and washed for 3 times, then resuspended with saline $(1 \mathrm{~mL})$. The similar process was applied for synthesis of NPs in different control groups.

\section{Characterization of IPPD NPs SEM/TEM}

The morphology of IPPD NPs and IPPD-PHF2 NPs was observed by TEM (HATACHI 7500, Japan) and goldsputtered SEM (ZEISS CrossBeam340, German). IPPDPHF2 NPs samples were prepared as follows: IPPD NPs $(100 \mu \mathrm{L}, 10 \mathrm{mg} / \mathrm{mL})$ was diluted to $1 \mathrm{mg} / \mathrm{mL}$ with doubledistilled Water (double distilled water) and added into plasmids solution $(100 \sim 150 \mu \mathrm{g} / \mathrm{mL}, 1 \mathrm{~mL})$, and then the mixture was incubated for $0.5 \mathrm{~h}$ at room temperature and centrifuged at the speed of $10000 \mathrm{rpm}$ for $8 \mathrm{~min}$. The sediment was re-suspended with double-distilled Water $(1 \mathrm{~mL})$.

\section{Standard Curves of IR780 and Dox}

IR780 $(2 \mathrm{mg})$ was dissolved in dimethylsulfoxide $(100 \mu \mathrm{L})$ and diluted to different concentrations with double-distilled Water. Samples were transferred to a 96-well plate (3 wells per sample), and the corresponding absorbance values at $780 \mathrm{~nm}$ were detected by automatic microplate reader (CYTATION5, BioTek). The standard curve of IR780 was calculated with GraphPad Prism 7. Similarly, the standard curve of Doxorubicin was accessed.

The entrapment efficiency and content were calculated by following Equations:

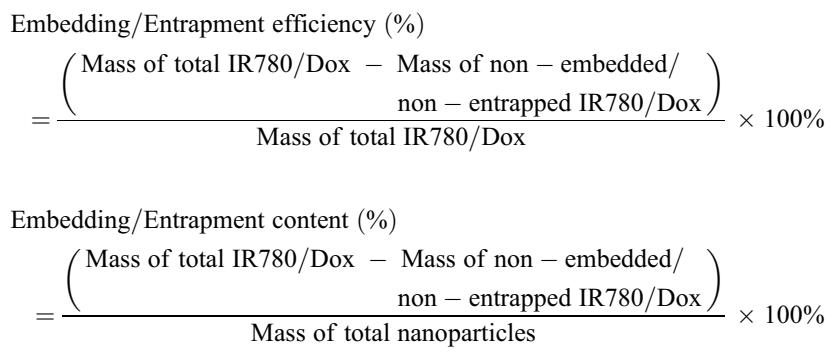

\section{In vitro Toxicity Test by CCK8 Assay}

MDA-MB-231 cells were seeded in 96-well plates (2000/ well). Then IPP NPs were diluted to different concentrations and added into wells (4 wells per concentration), except for the control group. Plate was washed for twice with sterile PBS after 24-hour incubation. CCK8 test was performed according to the protocol.

\section{FTIR Test}

PLGA (double-distilled Water as the inner phase) NPs and IPP NPs were synthesized and re-suspended with doubledistilled Water. Then they were freeze-dried into powders (FD-1A-80, BiLon co. Ltd., Shanghai) for FITR test.

\section{Co-Localization of IPPD NPs and Plasmids}

Nanoparticles were diluted with double-distilled Water $(1 \mathrm{~mL}, \quad 1 \mathrm{mg} / \mathrm{mL})$ and added in plasmid solution $(100 \sim 150 \mathrm{ng} / \mu \mathrm{L}, 400 \mu \mathrm{L})$ dyed with Gel-Red $(1 \mu \mathrm{L})$. The mixture was centrifuged (8min, 10000rpm). Sediment was gently re-suspended with double-distilled Water, and observed by LSCM (Nikon A1+, Japan). 


\section{Absorption Efficiency and Capacity}

IP NPs, IPD NPs, IPPD NPs were divided into five groups-0 (IP NPs),1(IPD NPs),2,3,4(IPPD NPs) $(1 \mathrm{mg} / \mathrm{mL}, 500 \mu \mathrm{L})$. Each sample was added in plasmids (40ug) dyed with GelRed. Group 0,1 were incubated for $60 \mathrm{~min}$ as control group, while group2,3,4 were incubated for $5 \mathrm{~min}, 10 \mathrm{~min}, 60 \mathrm{~min}$, respectively. Then samples were centrifuged and resuspended with double-distilled Water $(0.5 \mathrm{~mL})$. Resuspensions were detected by FCM (CytoFLEX), and supernatants were collected for plasmids concentrations detection (NanoDrop2000, Thermo Fisher Scientific, German).

\section{The Uptake Efficiency of IPPD NPs}

IPPD NPs and PPD NPs were synthesized and marked as experiment group and control group, respectively. MDA-MB -231 cells were seeded in LSCM dishes and marked as group $20 \mathrm{~min}, 40 \mathrm{~min}, 60 \mathrm{~min}, 120 \mathrm{~min}$. Both kinds of nanoparticles were re-suspended with cell culture medium $(0.1 \mathrm{mg} / \mathrm{mL})$ and dripped into dishes $(0.5 \mathrm{~mL})$. Dishes were washed with sterile PBS after prolonged incubation time. Cell membrane and nucleus were stained with DiI and DAPI, respectively. Samples were observed by LSCM under the same parameters. Operations were performed out of lights.

\section{In vitro Drug Release Test}

IPPD NPs (150mg, re-suspended with saline) were packed into preprocessed dialysis tubes and immersed into PBS $(100 \mathrm{~mL})$, the encapsulation efficiency was calculated according to the equations mentioned before (the mass of encapsulated Dox $=3.5352 \mathrm{mg}$, encapsulation efficiency $=39.28 \%$ ). The system was kept airtight and out of light in a shaker, and the samples were collected at different time points: $1 \mathrm{~h}, 4 \mathrm{~h}, 5 \mathrm{~h}$, $15 \mathrm{~h}, 25 \mathrm{~h}, 75 \mathrm{~h}$, and $172 \mathrm{~h}(600 \mu \mathrm{L}$ per sample). Samples were transferred to a 96 -well plate $(200 \mu \mathrm{L}$ per well), and detected for absorbance value at $490 \mathrm{~nm}$ by automatic microplate reader. The mass of released Dox was calculated according to the standard curve of Dox concentration. All operations were performed out of light.

\section{The Expression of EGFP, Western Blot and Wound Healing Assay}

Cells were digested and inoculated in LSCM dishes, a 6-well plate and T25 cell culture flasks. Transfection group was incubated with IPP-PHF2 (IR780/PLGA-PEIPHF2) NPs, while control group was treated with IPP NPs. On the basis of previous results of the safety nanoparticle concentration in vitro and the data of adsorption rate/ capacity of the nanoparticle, gene transfection in vitro was conducted according to the process below: since the normal standard of the mass of plasmids used in one well of a 6 -well plate is $4 \mu \mathrm{g}$, the mass was doubled to achieve a better result. The adsorption rate/capacity of nanoparticles reached approximately $80 \%$ and $65 \mu \mathrm{g} / \mathrm{mg}$ when the incubation lasted for $10 \mathrm{~min}$. Thus, $0.75 \mathrm{mg}$ nanoparticles were suspended in $1 \mathrm{~mL}$ saline, and added with over $48 \mu \mathrm{g}$ plasmids. With 10 15-minute incubation, the sediment after the mixture being centrifuged was re-suspended with $6 \mathrm{~mL}$ cell culture medium. Each of the three wells in transfection group was treated with $1 \mathrm{~mL}$ re-suspension. The nanoparticles which have not been mixed with plasmids were added into the control group at the same concentration and volume. The mass of plasmids used in T25 cell culture flasks were tripled compared to that used in the 6-well plate, whereas the mass of plasmids used in LSCM dishes was one-third of that used in the 6-well plate. The mediums containing nanoparticles were replaced by normal medium after 6 hours. The LSCM dishes were washed by PBS, then dyed by DiI and fixed by 4\% paraformaldehyde. These samples were observed under LSCM to verify the successful expression of the fusion gene EGFP. Flask samples were cultured for another three days, then collected for Western blot (PHF2, Vimentin, E-cadherin, CX43, ER $\alpha$, and $\beta$-actin, all operations were processed on the basis of WB protocol). Gray intensities were analyzed by ImageJ. For wound healing assay, scratches were formed with sterile tips after 2 days, and cells were washed with sterile PBS for 3 times, then the normal medium was replaced by low-serum medium $(1 \%)$. The observation of the widths of scratches continued for three days.

\section{Cell Apoptosis Assay}

Cells were seeded in 6-well plates, and marked as group 1, 2, 3, 4, 5, 6. Group1, 2, 3 and group 4, 5, 6 were treated with IPP-PHF2 NPs and IPP NPs, respectively. After 2-day incubation, group2, 5and group3, 6 were treated with Dox at concentrations of $0.0125 \mathrm{mg} / \mathrm{mL}$ and $0.0125 /$ $2 \mathrm{mg} / \mathrm{mL}$, respectively. Group1, 4 were control groups (without Dox). After three-day incubation, cell apoptosis rate was detected by FCM. EGFP fluorescence detection was applied to group1, 4.

\section{In vivo PA Imaging}

IPP NPs were diluted in saline to a concentration at $2 \mathrm{mg} / \mathrm{mL}$. PA images were captured via a Vevo LAZR Photoacoustic Imaging System (VisualSonics Inc., Toronto, Canada). 
Tumor-bearing nude mice were injected with IPP NPs solution $(200 \mu \mathrm{L})$ after the pre images were captured, and other PA images were obtained at different time points (2h, $4 \mathrm{~h}$ and 6h). Also, 770nm was defined and applied as the maximum absorption wavelength in vivo.

\section{In vivo Fluorescent Imaging}

Tumor-bearing mice were treated and imaged (Berthold NightOwl II LB 983-NC100) at the same conditions above. Other images were obtained at different time points (2h, $4 \mathrm{~h}$ and $6 \mathrm{~h}$ ), and the fluorescence intensities were analyzed by IndiGo.

\section{Cell Culture and MDA-MB-23। Tumor-Bearing Nude Mice Model}

Human breast cancer line MDA-MB-231 cells acquired from JXQ TECHNOLOGY (Chongqing, China) were cultured in high glucose medium (DMEM) complemented with $12 \%$ fetal bovine serum and $1 \%$ penicillin-streptomycin solution at cell incubator $\left(5 \% \mathrm{CO}_{2}, 37^{\circ} \mathrm{C}\right)$. Cells were sub-cultured in a proportion of $1: 2$ for both cell experiments and the construction of the sources for tumor block transplanting. Female nude mice and female Kunming mice (13-17g, 4-6w) were purchased from the Experimental Animal Center of CQMU (Chongqing Medical University). MDA-MB-231 cells were subcutaneously injected to the flanks of nude mice. The developed tumors were separated and cut into rice-grain-sized blocks for the following tumor block transplanting. All the experiments were approved by the Institutional Animal Care and Use Committee at CQMU and performed on the basis of the guideline of the Institutional Animal Care and Use Committee at CQMU. Tumor volumes were calculated by the following Equation:

$$
\text { c. } \mathrm{V}=(\text { length } \times \text { width } 2) / 2
$$

\section{Statistical Analysis}

All statistical analyses were conducted by GraphPad Prism 7 software. Data were presented as mean \pm standard deviation. The significance of the data is analyzed according to a Student's $t$-test: $* \mathrm{P}<0.05$.

\section{Results and Discussion}

\section{Synthesis and Characterization of IPPD-PHF2 Nanoparticles}

IR780 was embedded into PLGA nanoparticles, and Doxorubicin aqueous solution was encapsulated inside.
Then branched PEI was chemically conjugated to IPD NPs (IR780/PLGA (Dox)) via carbodiimide method to form a hull-shaped cationic nanoparticle (Figure 1A), considering that the carboxyl activated by EDC/sulfo-NHS can be used to aid the formation of peptide linkage between PLGA-COOH and branched PEI (containing rich $-\mathrm{NH}_{2}$ ). The ultimate nanoparticles possess welldefined spheral form and symmetrical size (Figure 2A). As presented in Figure 2B, the average size of IPPD-PHF2 nanoparticles in one sample was about $319.2 \mathrm{~nm}$, and the average size among different samples was $318 \pm 4.576 \mathrm{~nm}$. The average zeta potential of nanoparticles in one sample was stabilized at about $38 \mathrm{mV}$, and the average value of different samples was $37.87 \pm 0.4041 \mathrm{mV}$, as measured by DLS (Figure 2B). Absorbing ability of IPPD was tested by LSCM. For a better view, nanoparticles were gently resuspended into clustered particle blocks with larger size. Green represented Doxorubicin inside of the nanoparticles, while red was plasmids dyed by Gel-Red (this dye emits red fluorescence only when bonded with dsDNA), and a perfect co-localization was observed in the overlay image (Figure 2E). Visualized evidences were obtained by TEM/SEM. In the IPPD+Plasmids part, SEM image showed that plasmids (red arrows) were condensed and loaded onto the surface of IPPD (blue arrows), and the next was the corresponding TEM image. An apparent surface smoothness change was captured compared to the IPPD NPs part (Figure 2A). Also, the phenomenon that nanoparticles were semi-surrounded by plasmids was observed, from which both of the branched PEI hull and condensed plasmids could be seen, and more TEM images of NPs with various adsorption states were captured and displayed (Figure S1D). Zeta potential of PD, IPD, IPPD and IPPD-PHF2 NPs was measured (Figure 2B). Bar graph showed that the surface zeta potential increased after branched PEI was conjugated, and decreased when plasmids was loaded. Also, a comparison of differences among size, zeta potential and Polydispersity Index was presented, which were distinguished by nanoparticle types and IPPD nanoparticles with different levels of zeta potential. The results illustrated a positive correlation among zeta potential, size and PdI, which may be due to the mass of conjugated branched PEI (Figure S1A). FTIR test was conducted. The results illustrated specific peaks of amino bond in IPP NPs sample compared to the PLGA NPs sample (Figure 2F). According to the standard curve of IR780 at the wavelength of 780nm (Figure 2D), the mass of IR780 embedded in the nanoparticle was $0.0066 \mathrm{mg}$ per 

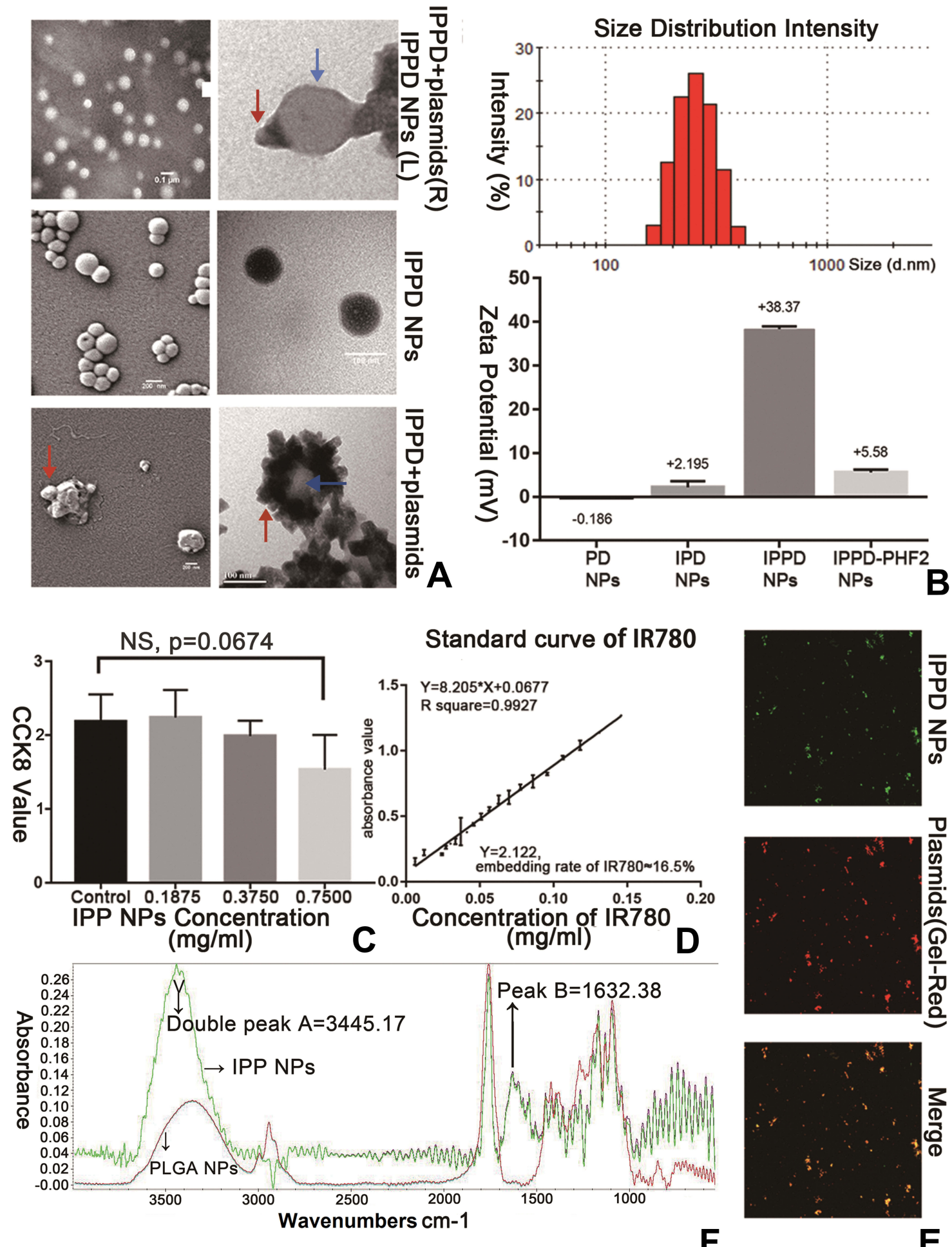

$\mathbf{F}$

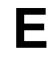

Figure 2 Characterization of the nanoparticles.

Notes: (A) SEM and TEM images of IPPD NPs and IPPD-PHF2 NPs (scale bars: TEM $=100 \mathrm{~nm}, \mathrm{SEM}=200 \mathrm{~nm}$ ). The red arrows represented the plasmids adsorbed onto the surface of IPPD NPs, while the blue arrows represented the IPPD NPs. (B) Size distribution of IPPD-PHF2 NPs and zeta potential of PD, IPD, IPPD and IPPD-PHF2 NPs. (C) Safety concentrations of IPP NPs. (D) Standard curve of IR780 concentration, regression equation: $Y=8.205 X+0.0677, R$ square $=0.9927$, embedding rate of IR780 $\approx 16.5 \%$. (E) Adsorption between IPPD NPs and PHF2, green: Doxorubicin, red: PHF2 dyed by Gel-Red. (F) Covalent binding between branched PEI and PLGA measured by FTIR, peak $A$ : the typical double peaks range from $3400 \mathrm{~cm}^{-1}$ to $3520 \mathrm{~cm}^{-1}$, peak B: the significant peak of primary amine in amido bond ranges from $1600 \mathrm{~cm}^{-1}$ to $1640 \mathrm{~cm}^{-1}$. 
milligram of nanoparticles. Safety dose of the nanoparticle was tested via CCK8 assay. Results yielded no significance between control group and $0.75 \mathrm{mg} / \mathrm{mL}$ group, and $0.75 \mathrm{mg} / \mathrm{mL}$ was much enough for our application (Figure 2C).

To further investigate adsorption efficiency and capacity of IPPD NPs, we compared the distribution ratio of NPs and the actual adsorption quantity of plasmids in different groups which were distinguished as 0-IP group, 1-IPD group, 2-5min-IPPD group, 3-10min-IPPD group, and 4-60min-IPPD group. All the groups, except for the control group 0 which was supposed to be double-fluorescencenegative, were mixed with equivalent plasmids (dyed with Gel-Red) and incubated for the corresponding time periods. The results of FCM indicated that almost all NPs in group 2,3,4 were covered with plasmid (Gel-Red+, Dox + ) (Figure 3A), while only a small percentage of NPs in group1 was double-fluorescence-positive which may be due to the positive potential of IR780 (Figure 2B). The determination of concentration results showed that the adsorption capacity of IPPD NPs had almost reached saturation after $10 \mathrm{~min}$ incubation, and it was about $64.804 \mathrm{ug} / \mathrm{mg}$ (Figure 3B).

Enhanced intracellular uptake efficiency was also observed by LSCM (Figure 3C). Under the same condition, MDA-MB-231 cells only showed low-intracellularuptake of PLGA-PEI (Dox) NPs after 2-hour incubation, while the fluorescence intensity of IPPD NPs at $20 \mathrm{~min}$ had already reached a much higher level. All in all, uptake efficiency has been significantly improved by IR780. The encapsulation efficiency of Doxorubicin was about $39.28 \%$ according to the standard curve (Figure 3D), and IPPD NPs presented a sustained drug release property (Figure 3E). Furthermore, we explored whether the intracellular uptake efficiency was uninfluenced or not when plasmids were loaded. LSCM results demonstrated perfect co-localization of plasmids and IPPD NPs and a timedependent enhanced uptake of the NPs (Figure S1B and $\underline{\mathrm{C}}$ ).

\section{Gene Transfection in vitro, Tumor Cells Undergoing MET Process, and Tumor-Inhibition Effect of PHF2}

More than $90 \%$ of cancer associated mortality is due to metastasis. During distant metastasis process, TICs (tumor initiating cells), containing the epithelial counterpart/the mesenchymal counterpart/the intermediates, must invade and pass through blood vessels, and circulate to the distant sites. When undergoing EMT, TICs de-differentiate into a more mesenchymal state. ${ }^{10,11}$ PHF2 is the hinge to induce more-mesenchymal counterparts of TICs into the epithelial state. $^{7}$ Herein, PHF2 was up-regulated by this nanoparticlebased transfection method. Tumor cells fluoresced bright green light, indicating successful co-expression of PHF2 and EGFP (Figure 4A). Western blotting showed that PHF2 in transfection group was significantly up-regulated compared to control group after 3-day incubation with IPPPHF2 NPs. Along with the over-expression of PHF2, E-cadherin was up-regulated, whereas Vimentin showed a descending tendency. E-cadherin belongs to the cadherin family - a sort of cell adhesion molecules (CAM) which is crucial in the formation of cell-to-cell adherence junctions. As a well-known symbol of epithelial cells, it has been reported that cells expressing a type of cadherin show tendency to cluster to other cell expressing the same type of cadherin. ${ }^{25,26}$ We also observed this phenomenon, cells in the transfection group tended to slowly grow into island clusters, while the non-transfection group in the same period gained great proliferation with equal distribution. Vimentin is expressed in mesenchyme-derived cells. It is the cytoskeletal component for the maintaining of cell integrity and shape. ${ }^{27,28}$ With the down-regulation of Vimentin, the pseudopodia of tumor cells became less obvious (Figure 5B).

To further verify the inhibited migration when tumor cells were undergoing MET, wound healing assay was performed. The results indicated that tumor cells stopped moving across to fill in the scratch when PHF2 was overexpressed. Equal amounts of IPP NPs and IPP-PHF2 NPs were added into control group and transfection group, respectively. After 2-day culture in 6-well plate, cells in each well were scratched with sterile tips, and cultured with low-serum culture medium (1\% FBS). The width of each scratch was measured. The widths of scratches in control group were narrowed from $140.3 \pm 2.309$ pixel to 76.33 \pm 1.155 pixel $(* * * p=0.0003)$, while the widths of scratches in transfection group changed from $102.3 \pm 3.055$ pixel to102.3 \pm 0.5774 pixel (ns, $\mathrm{p}>0.9999$ ) (Figure $5 \mathrm{~A}$ ).

When undergoing MET, not only the cell migration ability was inhibited, but also the sensitivity towards traditional chemotherapy is elevated. Thus, the proportions of tumor cells treated with different concentrations of Doxorubicin were detected by FCM (Figure 5D). The original concentration of Dox was selected from the effectiveness evaluation of Dox by CCK8 assay (Figure S2B). As shown in picture 

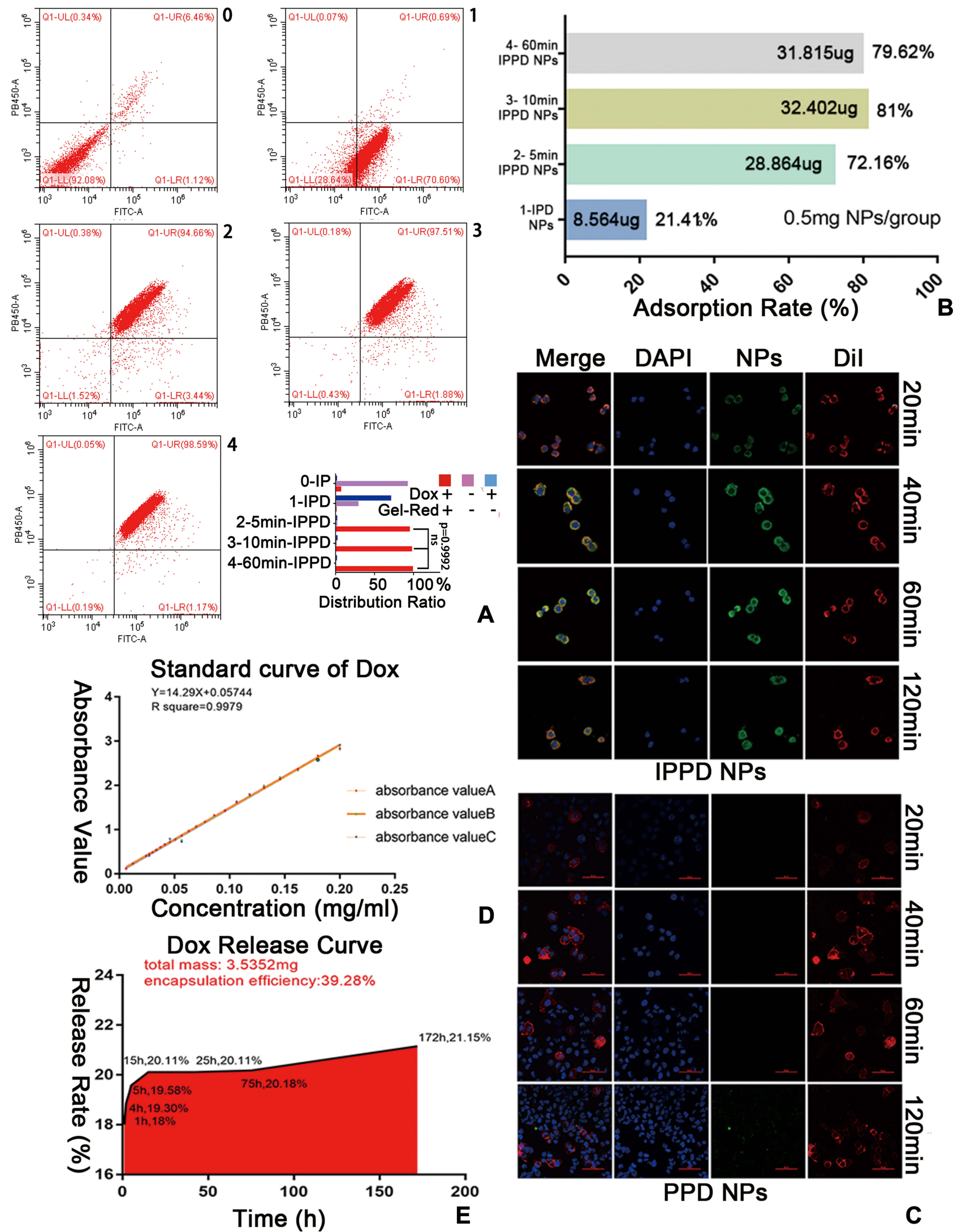

옹
3
3
․
옹
3
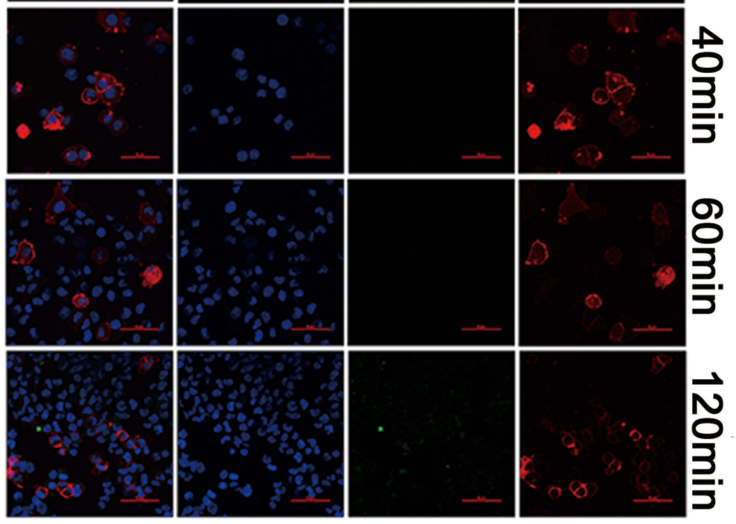

PPD NPs

$\stackrel{8}{3}$

C

Figure 3 Tests for properties.

Notes: (A) Adsorption rate of IPPD NPs detected by FCM, PHF2 were stained with Gel-Red, sample 0 and I were treated without PHF2 incubation. Sample 2, 3, 4 represented experimental groups incubated with PHF2 for $5 \mathrm{~min}, 10 \mathrm{~min}$ and $60 \mathrm{~min}$, respectively. (B) Quantification of adsorption capacity. (C) Comparison of NP-uptake between IPPD NPs and PPD NPs, bar scale=50um. (D) Standard curve of Dox concentration. (E) Drug release curve of IPPD NPs. 

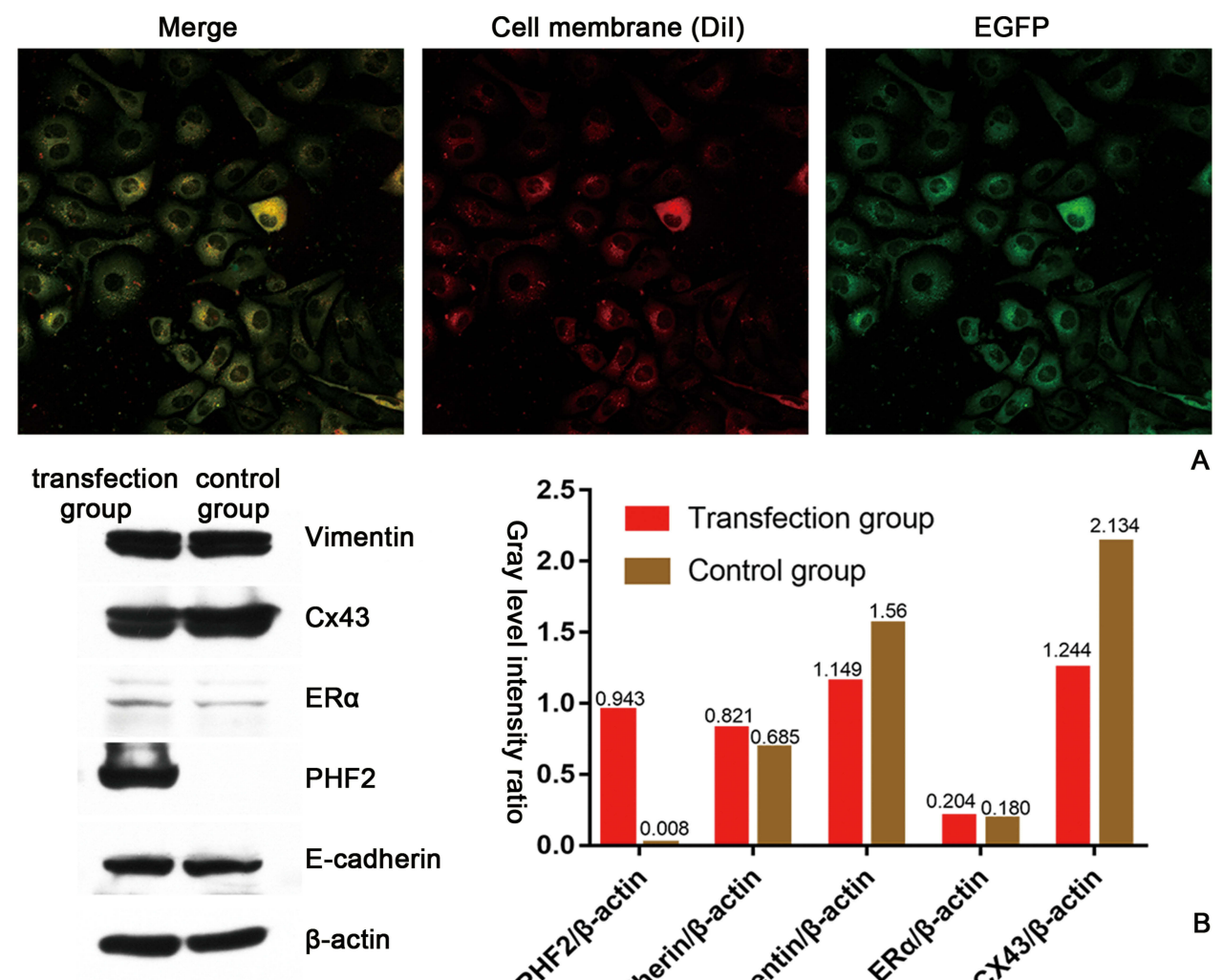

A
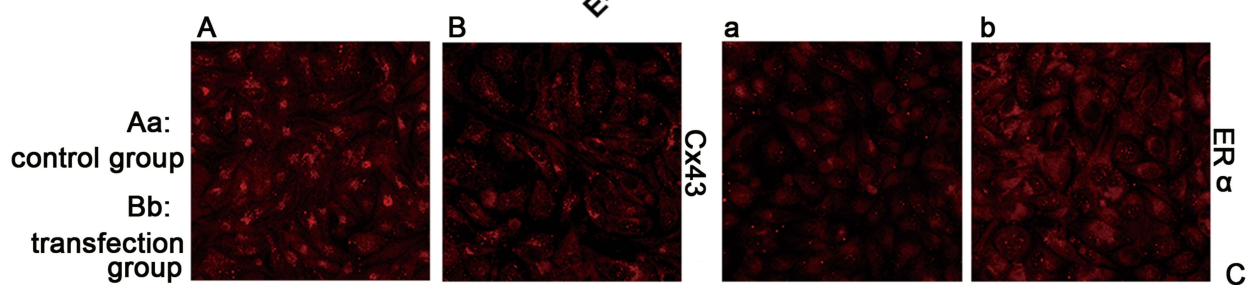

Figure 4 Gene transfection in vitro.

Notes: (A) Expression of EGFP after transfection. (B) Western Blotting results of expression level changes of PHF2 and related proteins after transfection, and the corresponding gray value intensity analysis. (C) Verification of expression level changes of ER $\alpha / C x 43$ by immunofluorescence.

1-EGFP and 4-EGFP (Figure 5D), about $88.62 \%$ of cells had detectable green fluorescence of EGFP in sample 1 which belonged to transfection group and was treated with no Doxorubicin, meanwhile, there were only $0.23 \%$ of cells had this fluorescence in sample4-EGFP, which was negligible. Equal amounts of IPP-PHF2 NPs and IPP NPs were added in transfection groups $(1,2,3)$ and control groups $(4,5,6)$, respectively. After incubation for 2 days, sample 2,5 were treated with Dox at the concentration of $0.0125 \mathrm{mg} /$ $\mathrm{mL}$, and sample 3, 6 were treated with half of the former concentration of Dox. And the results showed that the proportion of viable cells in control group was over 2-fold higher than the proportion in transfection group at both concentrations of Dox.

For in vitro gene transfection, Lipo2000 is the most common kit and can only be applied at cellular level. The comparison of cytotoxicity between Lipo2000 and IPP NPs was conducted. Cells in 96-well plate were treated with equal amounts of either Lipo2000 or IPP NPs at concentrations of 0 , $0.0625 / 16,0.00625 / 8,0.0625 / 4,0.0625 / 2$ and $0.0625 \mathrm{mg} / \mathrm{mL}$, and the cell viability was detected via CCK8 assay. The results indicated that there was no significance between the control group and the experiment group which was treated with the highest concentration of NPs, while a significant cytotoxicity was detected at the experiment group which was treated with Lipo2000 at the concentration of $0.0625 / 2 \mathrm{mg} / \mathrm{mL}$ ( $\mathrm{p}=0.0327$ ). IPP NP is safer than Lipo2000 (Figure 5C).

Gap junctional intracellular communication (GJIC) is generally related to tumorgenesis and progression. Studies focused on these connexins have reported so many controversial conclusions about the functions in cancers. However, more and more evidences indicated that connexins promote 

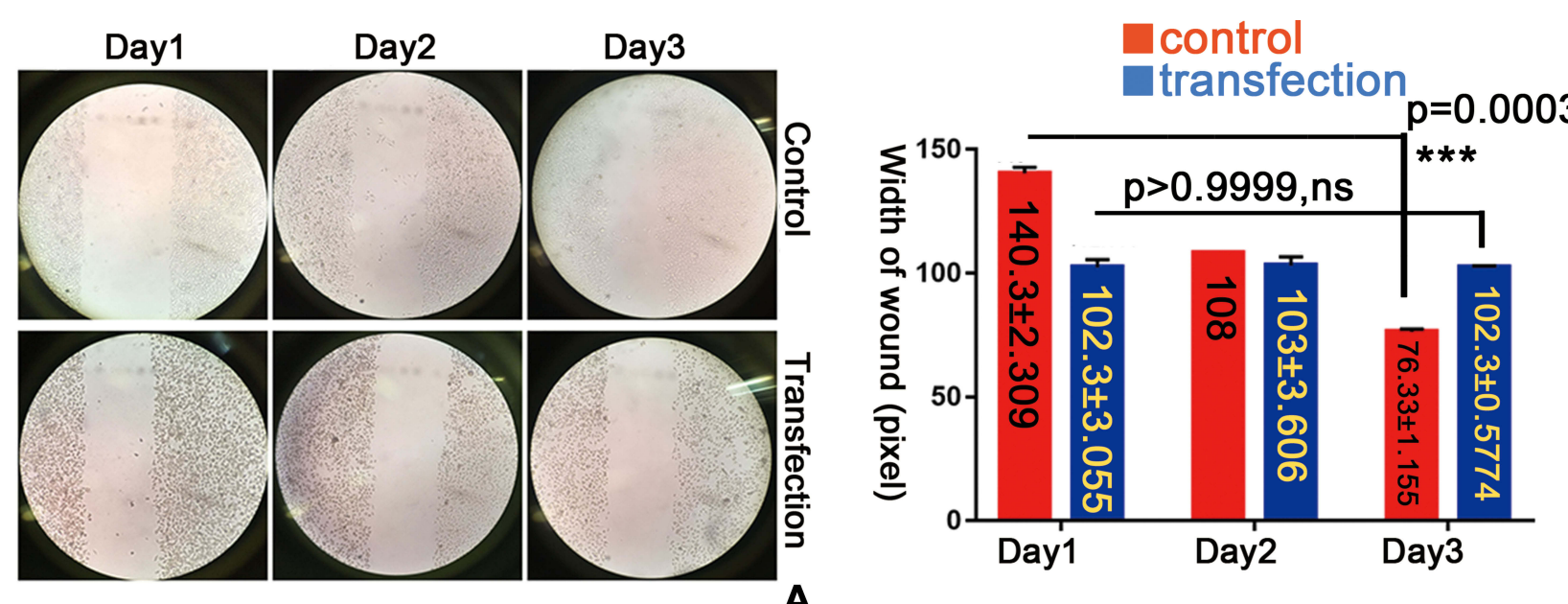

A

\section{Comparison of cytotoxicity}
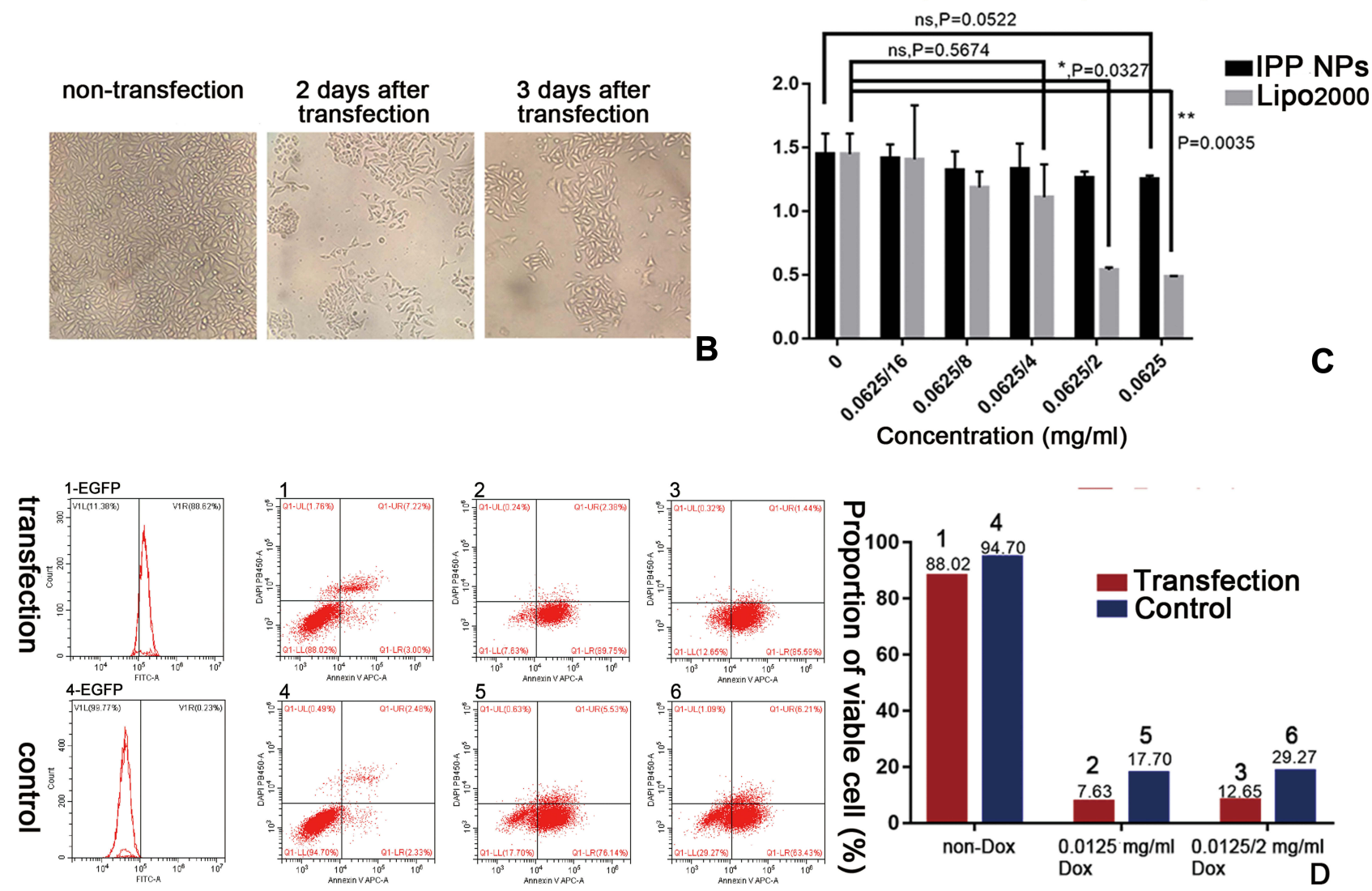

Figure 5 Anti-cancer effects of PHF2.

Notes: (A) Wound healing assay. The difference of width in transfection group between Day3 and Dayl showed no significance, $p>0.9999$, while the data in control group showed significance, $P=0.0003$. The widths of the wounds in day I and day 3 were compared by $t$-test. (B) Cell morphology change. (C) Comparison of cytotoxicity between IPP NPs and Lipo2000 via $t$-test (ns: non-significance, ${ }^{*} p<0.05,{ }^{*} p<0.01$, ${ }^{* * *} p<0.00$ I). (D) Enhanced drug sensitivity of MDA-MB-23I after transfection, I-EGFP and 4-EGFP were proportions of EGFP-expressing cells in groupl and group4, respectively.

tumor progression in specific tumor types and stage. Moreover, the activities of connexins are regulated by epigenetic regulation like DNA methylation. CX43, an universally expressed member of GJIC, confers temozolomide resistance and motility in glioblastoma multiforme through mitochondria apoptosis pathway. ${ }^{29,30}$ Given that, we explored the expression level of CX43 when PHF2 was overexpressed. Results indicated that CX43 and PHF2 were negatively related (Figure 4B and C). According to previous conclusions, lowered expression of CX43 may contribute to 


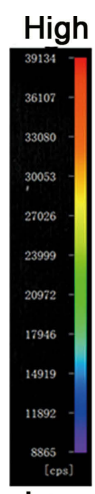

Low
Pre

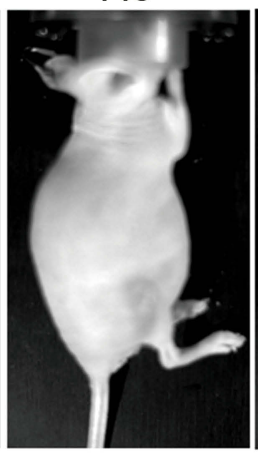

$2 h$

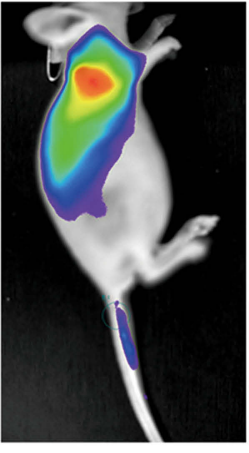

$4 h$

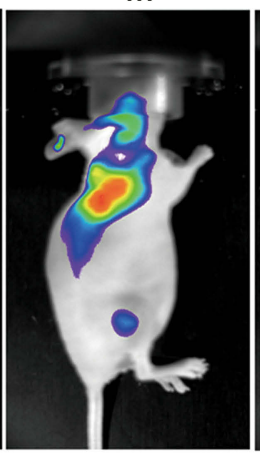

$6 h$

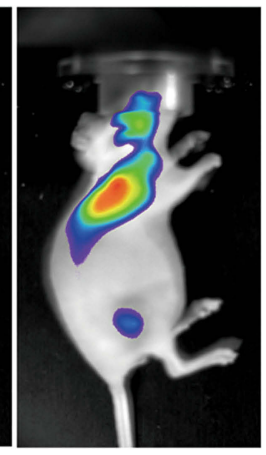

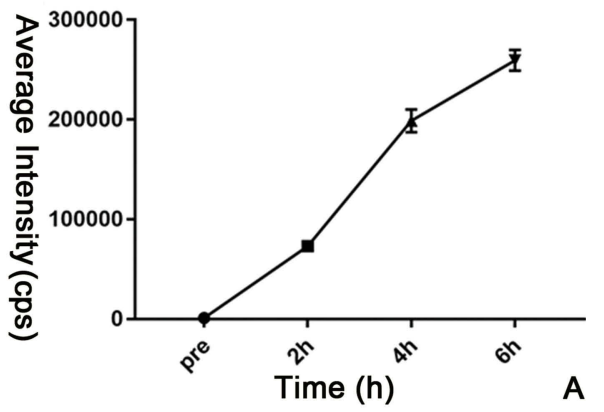

Low Pre

$2 \mathrm{~h}$

$4 h$

$6 \mathrm{~h}$
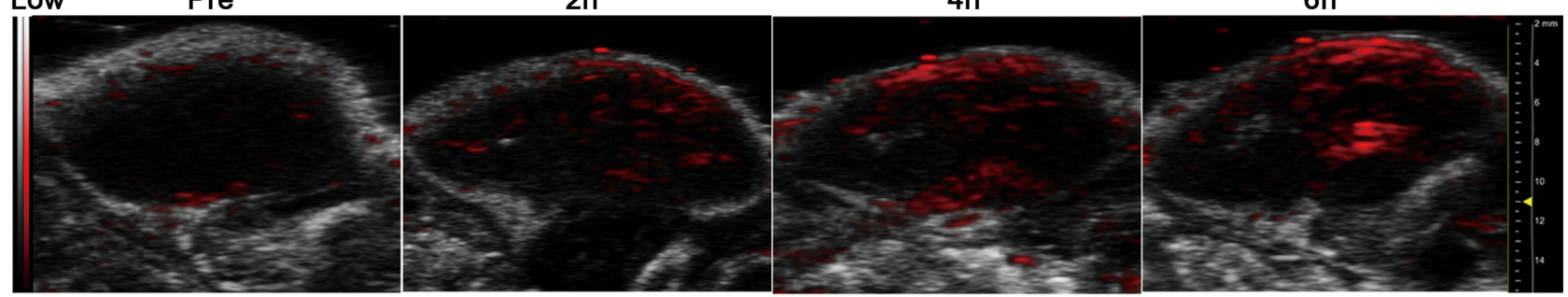

High

Figure 6 Multimodality imaging.

Notes: (A) Fluorescent Imaging. (B) Photoacoustic Imaging.

drug resistance and distant metastasis caused by PHF2overexpression. A more thorough exploration is in progress.

$\mathrm{ER} \alpha$ has been reported to inhibit breast cancer metastasis and extend life span of mice when up-regulated in MDA-MB-231 and MCF-7 models. Furthermore, the enhanced formation of amoeboid-like protrusions (such as pseudopods and blebs) was observed in Cas9-ER $\alpha$ MCF-7 cells, which indicated that invasion and metastasis were promoted. Suppression effects of ER $\alpha$ in cellular motility/invasion by inhibiting EMT were reported. ${ }^{31}$ Given that, ER $\alpha$ has come in sight with the doubt-whether it is related to the PHF2-induced inhibition of metastasis. Despite the weakly positive ER $\alpha$ expression caused by nonspecific binding, results of Western Blotting and immunofluorescence illustrated that $\mathrm{ER} \alpha$ was upregulated when PHF2 was over-expressed. Morphology change was in consistent with the mentioned protrusion changes (Figure 5B). Despite this uncertain relation of $\mathrm{ER} \alpha$ in tumor metastasis, its up-regulation has provoked us of an auxiliary endocrinotherapy. Though the final effect of PHF2-upregulation is anti-cancer, the direct/indirect up-regulation of $\operatorname{ER} \alpha$ may have some negative impacts against the anti-cancer efficiency of PHF2 in proliferation. Endocrinotherapy-related study is yet to be done. ${ }^{34}$

\section{Tumor-Targeting in vivo Observed by Fluorescence Imaging, PA Imaging}

Photoacoustic Imaging is based on photoacoustic effect-thermoelastic waves generated by the absorption of electromagnetic energy (EM) such as optical waves have conquered both the weak contrast of ultrasound Imaging and poor resolution of EM Imaging. Non-ionizing EM Imaging poses no health threat. Ultrasound Imaging is a detection of waves reflected off body structure, which possesses better resolution than optical imaging. However, low contrast of Ultrasound Imaging is considered to be a crucial shortcoming, which can be overcome by high contrast of EM Imaging. Visible (400-700nm) and infrared regions (700-1100nm) of EM spectrum are inherently sensitive to abnormal tissues and have good tissue penetration abilities. ${ }^{22,32}$

MDA-MB-231 tumor-bearing mice were injected with IPP NPs $(2 \mathrm{mg} / \mathrm{mL}, 200 \mu \mathrm{L})$ after the pre-PA images were captured. $770 \mathrm{~nm}$ was defined as the maximum absorption wavelength in vivo. Images were obtained at different time points ( $2 \mathrm{~h}, 4 \mathrm{~h}$ and $6 \mathrm{~h}$ ). The intensity of PA signal showed a time-dependent tendency (Figure 6B).

In vivo NIR Fluorescence Imaging is considered as a functional imaging mode with better tissue penetration, 
high sensitivity, non-invasiveness and non-ionizationradiation. It is safer, cheaper, more intuitionistic and easier to operate. ${ }^{33}$ The autofluorescence/tumor-targeting characteristics of IR780 extended the nanoparticles with specific tumor fluorescence imaging at the near-infrared region $(\lambda$ excitation $/ \lambda$ emission $=770 \mathrm{~nm} / 810 \mathrm{~nm})$. The fluorescence images were captured at elongated time points after injection of IPP NPs, and signal intensity was enhanced in a time-dependent manner (Figure 6A).

\section{In vivo Epigenotherapy and Synergistic Chemotherapy by IPPD-PHF2 Nanoparticles}

MDA-MB-231 tumor-bearing nude mice were divided into seven groups as follows: i.v. saline, IPPD-PHF2 NPs, IPPPHF2 NPs, IPPD NPs, PP-PHF2 NPs (PLGA-PEI-PHF2), i.v. PHF2, IPP NPs group. Each group was injected with the corresponding content $(2 \mathrm{mg} / \mathrm{mL}, 200 \mu \mathrm{L})$ at dayl, day8, day14, and the tumor size and mice weight were recorded. After 20 days, tissues (heart, liver, spleen, lung, kidney, tumor) were collected for further tests. Among these tissues, the expression levels of PHF2/E-cadherin/ Vimentin in liver/lung/tumor were detected via IHC. Apoptotic cells and proliferative cells were detected by immunofluorescence staining of PCNA and TUNEL test.

$H \& E$ staining results have illustrated that 1.Various degrees of karyopyknosis were observed in IPP-PHF2 NPs group and IPPD-PHF2 NPs group. In IPPD-PHF2 NPs group, a large proportion of nucleus simultaneously presented karyopyknosis, karyorrhexis and karyolysis, while high nuclear-cytoplasmic ratio remained in other groups. 2. In IPPD-PHF2 NPs group and IPP-PHF2 NPs group, structures of liver and lung were normal and no metastasis was found (Figure 8A). To avoid false-negative results, tissues were observed by serial sections (Figure S4). Apparent structure damages were observed in other groups: hepatic lobules and pulmonary alveoli lost the proper histomorphology, and central veins/hepatic sinusoidal/bronchi were occupied by masses of heterotypic cells. Heart/spleen/ kidney presented normal histomorphology among all groups. These results indicated that the synergistic effect between PHF2 and Doxorubicin caused a stronger inhibition in DNA transcription than each single action, which contributed to the prohibited tumor growth and migration. Immunofluorescence staining of PCNA and TUNEL tests further confirmed this effect. A holistic view of tumor section of IPPD-PHF2 NPs group demonstrated a high ratio of cell apoptosis (TUNEL, green) and an extremely low ratio of cell proliferation (PCNA, red), while other groups presented reverse results. Statistical results of PCNA/TUNEL were calculated by cell-counting in three randomly selected areas of tumor sections (Figures $8 \mathrm{~B}$ and $\underline{\mathrm{S} 2}$ ). In IPPD-PHF2 NPs group, nearly half cells ( $\approx 49.54 \%)$ appeared to be TUNEL-positive, while almost none of the cells $(\approx 0.97 \%)$ presented PCNA-positive. The ratios of PCNA-positive cells were $(\approx 60.31 \%)$ and $(\approx 6.47 \%)$ in IPPD NPs group and IPP-PHF2 NPs group, respectively, whereas the ratios of TUNEL-positive cells were basically flat $(6.59 \%, 0 \%$, respectively) and much lower than that in IPPD-PHF2 NPs group, implicating Doxorubicin along with PHF2 generated a synergistic effect on anti-cancer behaviors. In addition, cells in PP-PHF2 NPs group presented higher PCNA-positive ratio compared to cells in IPP-PHF2 group, which was correspondent with the expression levels of PHF2 in IHC results, implying a weaker transfection efficiency in vivo (Figure 9A).

Immunohistochemistry tests (IHC) for detecting the expression levels of PHF2/E-cadherin/Vimentin were implemented on tumor/liver/lung. According to the data in NCBI database, expression levels of PHF2 in normal tissues appear to be high in lung, secondarily in breast, then in liver (Figure S2A). However, it is down-regulated in breast cancer cells. In IPPD-PHF2 NPs group and IPPPHF2 NPs group, shades of brown localized in nucleus were observed in most of cells, while only a small proportion of nucleus-localized light brown was detected in PPPHF2 NPs group and the rest groups presented negative results, which illustrated that PHF2 was obviously upregulated and IPP NPs facilitated transfection in a more efficient way comparing to PP NPs. As expected, the membranous/cytoplasmic expression levels of E-cadherin and Vimentin were aligned with cell experiment results, which signified successful induction of MET in vivo (Figure 9A). Given the flat expression levels of PHF2 in liver and lung between the IPP-PHF2 NPs group and normal nude mice, well-targeted transfection was presented in vivo (Figure 9B).

Tumor width and length were recorded at day1, day3, day6, day8, day11, day14, day17, day20. After 20 days, average volume of tumors in IPPD-PHF2 NPs group remained flat compared to that in day1, whereas $3 \sim 10$-fold increase were observed in other groups (Figure 7A). Meanwhile, no significant change in weight was found among groups during the whole treating process (Figure 7B). 

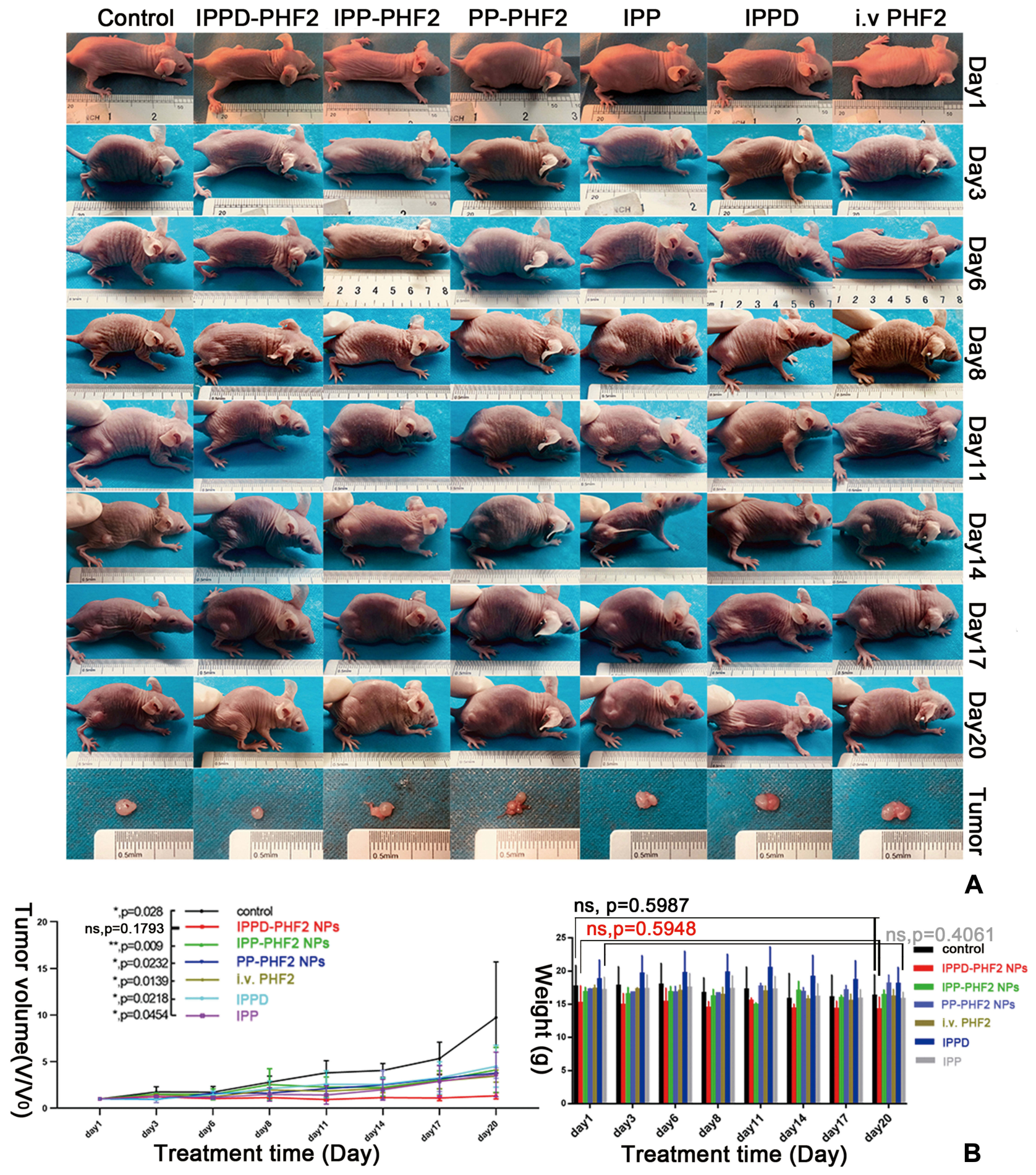

Figure 7 Anti-cancer effects in vivo.

Notes: (A) Photos of MDA-MB-23I tumor-bearing nude mice captured during the 20-day treating process and photos of tumors at the end of treatments. (B) Tumor growth curve and weight changes of the mice. The difference of tumor volume between Day 20 and Dayl in the IPPD-PHF2 group showed no significance, $\mathrm{P}=0.1793$, while in other groups, the difference was significant at various levels. The difference of mice weight between Day20 and Dayl in control group, IPP group and IPPD-PHF2 group showed no significance, the $\mathrm{p}$-value was $0.5987,0.406 \mathrm{I}$, and 0.5948 , respectively. Data in day 20 and dayl were compared by $t$-test (ns: non-significance, ${ }^{*} \mathrm{p}<0.05$, $* * \mathrm{p}<0.0 \mathrm{I}$ ).

\section{In vivo Biosafety of IPP NPs}

Female Kunming mice were used for serological tests to confirm the safety application of IPP NPs in vivo. No significant changes were found among the blood routine examination results and blood biochemistry results of group 1-day, group3-day, group 5-day, and group non-i.v. 

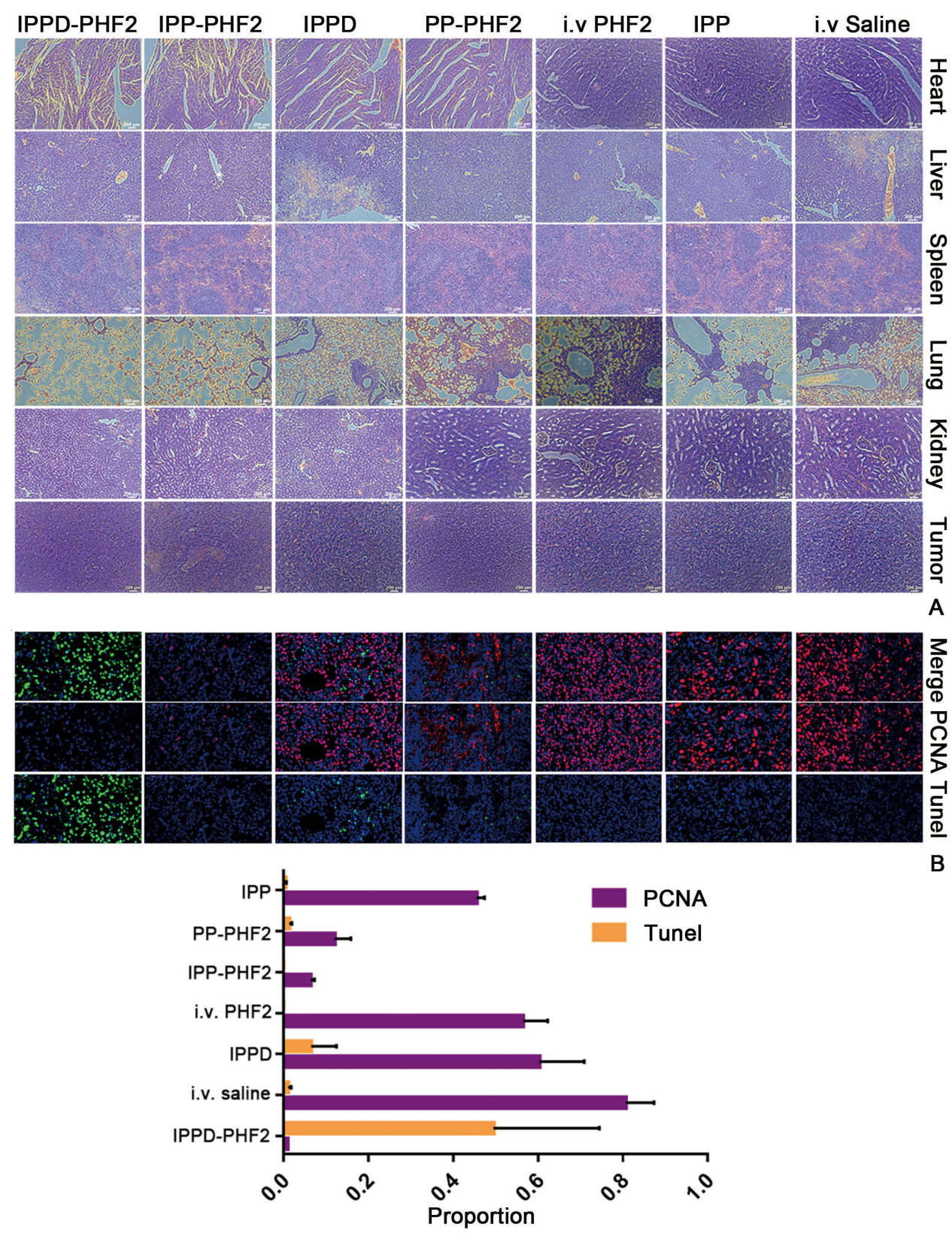

Figure 8 Histology detection of tumor tissue.

Notes: (A) H\&E staining. (B) PCNA and TUNEL.

Herein, IPP NPs were safe for in vivo application (Figure $\underline{\mathrm{S} 3 \mathrm{~A}}$ and $\underline{\mathrm{B}})$.

\section{Conclusions}

In general, we have properly validated our previous hypothesis that the up-regulation of PHF2 is actually a promising choice for epigenotherapy. IPPD-PHF2 was applied to implement targeted epigenotherapy based on PHF2upregulation which induced tumor cells to undergo MET. With a significant up-regulation of PHF2, MDA-MB-231 breast tumor cells phenotypically changed from a malignant type to a more benign type, and became more vulnerable against chemotherapeutic drug and less metastatic. Along with the successful transfection, Doxorubicin, as an activator of p53, was slowly released. Meanwhile, PHF2, a necessary factor in p53-p21 pathway which causes anticancer effects, co-activated with Dox, further amplified the chemotherapeutic effects (Figure S5). All these changes and anti-cancer effects specifically occurred in tumor cells due to the extraordinary tumor-targeting ability of IR780 which 


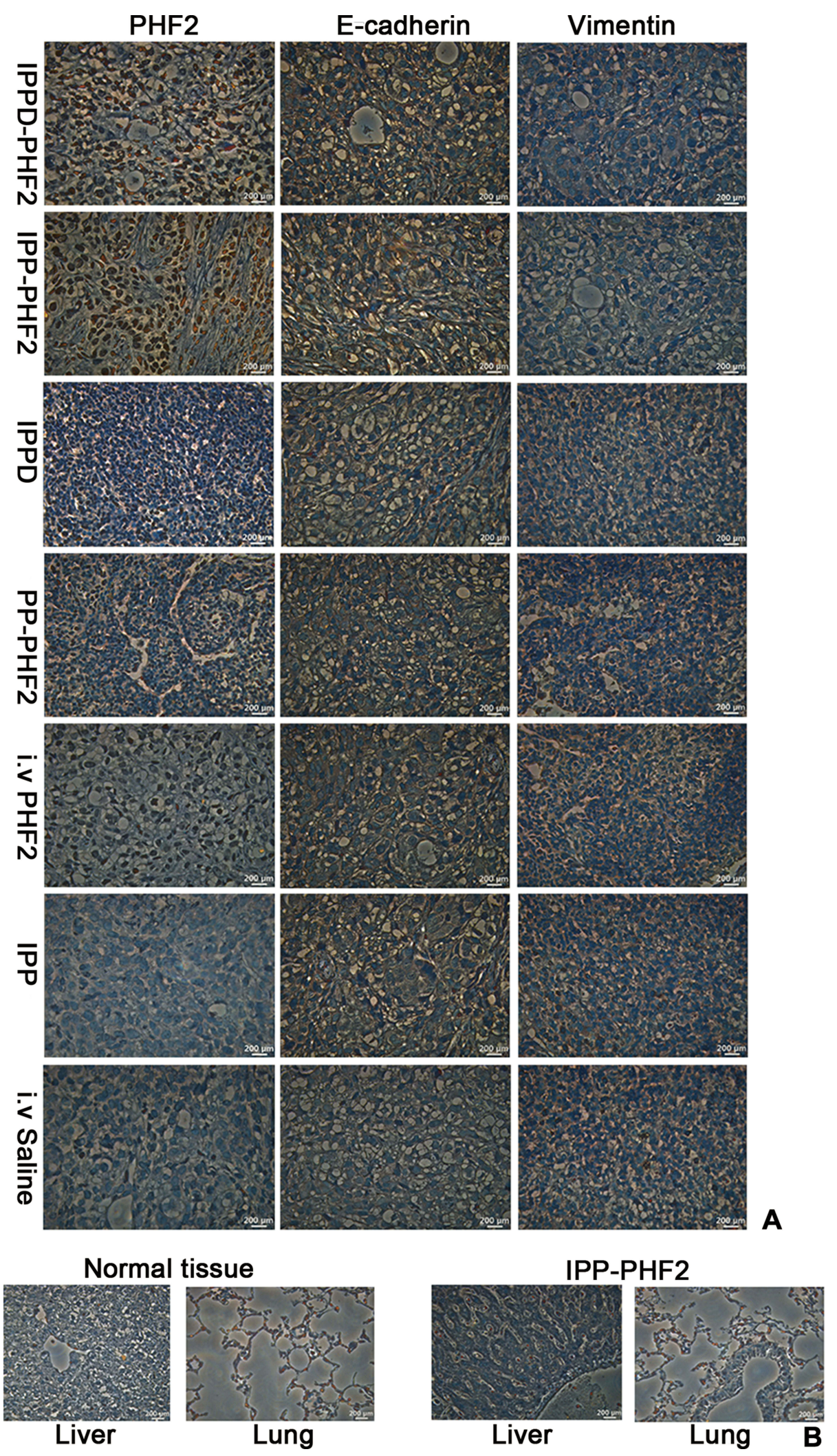

Figure 9 Immunohistochemical detection.

Notes: (A) IHC results of PHF2/E-cadherin/Vimentin expression in tumor tissues. (B) Comparison of PHF2 expression levels in liver/lung between normal nude mice and nude mice treated with IPP-PHF2 NPs. 
greatly improved transfection efficiency and precision, as shown in the Fluorescent/Photoacoustic Imaging results: nanoparticles accumulated in tumor sites specifically and rapidly in a time-dependent manner. Furthermore, the other characteristics, as to say the NIR fluorescence and photosensitivity, rendered IPPD-PHF2 NPs to sequentially track tumors during the treating process.

In this study, we explored a tumor-targeting nanoparticle to utilize metastasis-inhibiting epigene and amplified strikes of chemotherapeutic to treat, and conduct multimodality imaging to locate. It is considerable to go further exploring the hidden capacity of tumor-targeting PHF2epigenotherapy. What we have preliminarily validated about the related changes of ER $\alpha$ and CX43 posed hints to us. Up-regulation of ER $\alpha$ provides feasibility to perform endocrinotherapy and thus eliminate its negative effects, which may amplify the anti-cancer ability, so-called "triple therapy". ${ }^{34}$ Also, inhibiting the activity of CX43 may also reduce metastasis, regarding its GJIC role between tumor cells.

Except for the subline therapeutic possibilities of IPPD-PHF2 NPs, the main course which shall be the promotion of PHF2 activation is guiding us to the maximization of the anti-cancer effects. As a demethylase, PHF2 is known as a PKA-dependent downstream factor by now. Recovering its anti-cancer role by altering the deleted state in tumor cells and the phosphorylation effect of PKA pathway have limitations. Thus, a direct non-PKA -dependent activation of PHF2 comes up as a choice, which means the discovery of an activator aiming to cast enzymatic reaction and directly phosphorylate PHF2 is of great value. Combination of PHF2-epigenotherapy, Dox chemotherapy, non-PKA-dependent activation of PHF2 may bring us significant therapeutic effects.

With these advantages and perspectives, IPPD-PHF2 may be an epigene-drug-combined system with great prospects in cancer theranostics.

\section{Acknowledgments}

This work was supported by the National Natural Science Foundation of China [Grant Nos. 31630026, 81630047, 81601513]; Basic Research and Frontier Exploration of Chongqing Science and Technology Commission [Grant Nos. cstc2018jcyjAX0747 and cstc2019jscx-dxwtBX0004]. Appreciation for technical support on SEM from Prof. Zhong Yang of Third Military Medical University (Grant No. 31571242).

\section{Disclosure}

Mengzhu Wang, Zhigang Wang, Hanwen Qi and Xianquan Zhang report a patent related to this work, number 201811520731.3. The authors report no conflicts of interest in this work.

\section{References}

1. Bannister AJ, Kouzarides T. Regulation of chromatin by histone modifications. Cell Res. 2011;21:381-395. doi:10.1038/cr.2011.22

2. Højfeldt JW, Agger K, Helin K. Histone lysine demethylases as targets for anticancer therapy. Nat Rev Drug Discov. 2013;12:917-930. doi: $10.1038 / \mathrm{nrd} 4154$

3. Wen H, Li J, Song T, et al. Recognition of histone H3K4 trimethylation by the plant homeodomain of PHF2 modulates histone demethylation. J Biol Chem. 2010;285:9322-9326. doi:10.1074/jbc. C109.097667

4. Mozzetta C, Boyarchuk E, Pontis J, et al. Sound of silence: the properties and functions of repressive Lys methyltransferases. Nat Rev Mol Cell Biol. 2015;16:499-512. doi:10.1038/nrm4029

5. Hyun K, Jeon J, Park K, et al. Writing, erasing and reading histone lysine methylations. Exp Mol Med. 2017;49:1-22.

6. Lee KH, Park JW, Sung HS, et al. PHF2 histone demethylase acts as a tumor suppressor in association with p53 in cancer. Oncogene. 2014;34:2897-2909. doi:10.1038/onc.2014.219

7. Pattabiraman DR, Bierie B, Kober KI, et al. Activation of PKA leads to mesenchymal-to-epithelial transition and loss of tumor-initiating ability. Science. 2016;351:aad3680. doi:10.1126/science.aad3680

8. Shi G, Wu M, Fang L, et al. PHD finger protein 2 (PHF2) represses ribosomal RNA gene transcription by antagonizing PHF finger protein 8 (PHF8) and recruiting methyltransferase SUV39H1. J Biol Chem. 2014;289:29691-29700. doi:10.1074/jbc.M114.571653

9. Kooistra SM, Helin K. Molecular mechanisms and potential functions of histone demethylases. Nat Rev Mol Cell Biol. 2012;13:297-312. doi:10.1038/nrm3327

10. Nieto MA, Huang RY, Jackson RA, et al. EMT: 2016. Cell. 2016;166 (1):21-45. doi:10.1016/j.cell.2016.06.028

11. Brabletz T. EMT and MET in metastasis: where are the cancer stem cells? Cancer Cell. 2012;22:699-701. doi:10.1016/j.ccr.2012.11.009

12. Yeh PY, Chuang SE, Yeh KH, et al. Phosphorylation of p53 on Thr55 by ERK2 is necessary for doxorubicin-induced p 53 activation and cell death. Oncogene. 2004;23:3580-3588. doi:10.1038/sj.onc. 1207426

13. Stern M, Ulrich K, Geddes DM, et al. Poly (D, L-lactide-coglycolide)/DNA microspheres to facilitate prolonged transgene expression in airway epithelium in vitro, ex vivo and in vivo. Gene Ther. 2003;10:1282-1288. doi:10.1038/sj.gt.3301994

14. Panyam J, Labhasetwar V. Biodegradable nanoparticles for drug and gene delivery to cells and tissue. Adv Drug Deliv Rev. 2012;55:1-11.

15. Chung HJ, Kim HK, Yoon JJ, et al. Heparin immobilized porous PLGA microspheres for angiogenic growth factor delivery. Pharm Res. 2006;23:1835-1841.

16. Panyam J, Zhou WZ, Prabha S, et al. Rapid endo-lysosomal escape of poly(DL-lactide-coglycolide) nanoparticles: implications for drug and gene deliver. FASEB J. 2002;16:1217-1226. doi:10.1096/fj.02$0088 \mathrm{com}$

17. Astete CE, Sabliov CM. Synthesis and characterization of PLGA nanoparticles. $J$ Biomater Sci Polym Ed. 2006;17:247-289. doi:10.1163/156856206775997322

18. Wang D, Robinson DR, Kwon GS, et al. Encapsulation of plasmid DNA in biodegradable poly(D,L-lactic-coglycolic acid) microspheres as a novel approach for immunogene delivery. $J$ Control Release. 1999;57:9-18. doi:10.1016/S0168-3659(98)00099-6 
19. Kasturi SP, Sachaphibulkij K, Roy K. Covalent conjugation of polyethyleneimine on biodegradable microparticles for delivery of plasmid DNA vaccines. Biomaterials. 2005;26:6375-6385. doi:10.1016/j. biomaterials.2005.03.043

20. Bivas-Benita M, Romeijn S, Junginger HE, et al. PLGA-PEI nanoparticles for gene delivery to pulmonary epithelium. EUR J Pharm Biopharm. 2004;58:1-6. doi:10.1016/j.ejpb.2004.03.008

21. Alves CG, Lima-Sousa R, de Melo-diogo D, et al. IR780 based nanomaterials for cancer imaging and photothermal, photodynamic and combinatorial therapies. Int J Pharm. 2018;542:164-175. doi:10.1016/j.ijpharm.2018.03.020

22. Yang X, Shi C, Tong R, et al. Near infrared heptamethine cyanine dye-mediated cancer imaging. Clin Cancer Res. 2010;16:1-21. doi:10.1158/1078-0432.CCR-10-0059

23. Tan X, Luo S, Wang D, et al. A NIR heptamethine dye with intrinsic cancer targeting, imaging and photosensitizing properties. Biomaterials. 2012;33:2230-2239. doi:10.1016/j.biomaterials.2011.11.081

24. Guo F, Yu M, Wang J, et al. The mitochondria-targeted and IR780-regulated theranosomes for imaging and enhanced photodynamic/photothermal therapy. RSC Adv. 2016;6:11070-11076. doi:10.1039/C5RA19521G

25. Wong AS, Gumbiner BM. Adhesion-independent mechanism for suppression of tumor cell invasion by E-cadherin. J Cell Biol. 2003;161:1191-1203. doi:10.1083/jcb.200212033
26. Beavon IR. The E-cadherin-catenin complex in tumour metastasis: structure, function and regulation. Eur J Cancer. 2000;36:1607-1620. doi:10.1016/S0959-8049(00)00158-1

27. Fuchs E, Weber K. Intermediate filaments: structure, dynamics, function, and disease. Annu Rev Biochem. 1994;63:345-368. doi:10.1146/ annurev.bi.63.070194.002021

28. Ulirsch J, Fan C, Knafl G, et al. Vimentin DNA methylation predicts survival in breast cancer. Breast Cancer Res Treat. 2013;137:383-396. doi:10.1007/s10549-012-2353-5

29. Gielen PR, Aftab Q, Ma N, et al. Connexin43 confers Temozolomide resistance in human glioma cells by modulating the mitochondrial apoptosis pathway. Neuropharmacology. 2013;75:539-548. doi:10.1016/j. neuropharm.2013.05.002

30. Aasen T, Mesnil M, Naus CC, et al. Gap junctions and cancer: communicating for 50 years. Nat Rev. 2016;16:775. doi:10.1038/nrc.2016.105

31. Gao Y, Wang Z, Hao Q, et al. Loss of ER $\alpha$ induces amoeboid-like migration of breast cancer cells by downregulating vinculin. Nat Commun. 2017;8:1-15. doi:10.1038/ncomms14483

32. Xu M, Wang LV. Photoacoustic imaging in biomedicine. Rev Sci Instrum. 2006;77:041101. doi:10.1063/1.2195024

33. Frangioni JV. In vivo near-infrared fluorescence imaging. Curr Opin Chem Biol. 2003;7:626-634. doi:10.1016/j.cbpa.2003.08.007

34. Schiff R, Massarweh S, Shou J, et al. Breast cancer endocrine resistance: how growth factor signaling and estrogen receptor coregulators modulate response. Clin Cancer Res. 2003;9:447S-54S.
International Journal of Nanomedicine

\section{Publish your work in this journal}

The International Journal of Nanomedicine is an international, peerreviewed journal focusing on the application of nanotechnology in diagnostics, therapeutics, and drug delivery systems throughout the biomedical field. This journal is indexed on PubMed Central, MedLine, CAS, SciSearch ${ }^{\mathbb{}}$, Current Contents ${ }^{\mathbb{R}} /$ Clinical Medicine,
Journal Citation Reports/Science Edition, EMBase, Scopus and the Elsevier Bibliographic databases. The manuscript management system is completely online and includes a very quick and fair peer-review system, which is all easy to use. Visit http://www.dovepress.com/ testimonials.php to read real quotes from published authors. 